\title{
A Revolução dos Lasers de Femtossegundo na Oftalmologia
}

$\mathbf{O}$ primeiro laser (Light Amplification by Stimulated Emission of Radiation Light - ampliação de luz por meio da emissão estimulada de radiações) foi desenvolvido pelo físico americano Theodore H. Maiman em 1960 com cristal de rubi ${ }^{(1)}$, dando continuidade aos estudos pioneiros de Albert Einstein relacionados com a Teoria Quântica da Radiação. Apesar das importantes limitações relacionadas com a não estabilidade e da duração do pulso inadequada deste primeiro laser de rubi, o trabalho de Maiman pode ser considerado como a epifania que abriu caminho para impressionante evolução desta fantástica forma de energia para diversas aplicações na Medicina.

A Oftalmologia se destaca por ter sido a primeira especialidade na Medicina a utilizar o laser com objetivo terapêutico ${ }^{(2)}$, bem como pelo seu acelerado e constante desenvolvimento. De fato, o termo "laser" apresenta uma conotação de tecnologia avançada e futurística. Mas devemos entender que, como os computadores, os lasers estão em uma constante e rápida evolução, o que possibilita aplicações diagnósticas e terapêuticas cada vez mais precisas e eficazes. Diversos tipos de laser como argônio, criptônio, dióxido de carbono, neodímio-YAG (yttrium aluminum garnet [Nd:YAG]) e excimer foram desenvolvidos para diversas aplicações na Oftalmologia. Os diferentes lasers são caracterizados pelo comprimento de onda dentro do espectro eletromagnético da luz. Este é determinado pela fonte do laser e pelo meio ativo, sendo definido pela distância que o fóton percorre enquanto o campo elétrico completa uma oscilação. As características e formas de interação laser-tecido - fotocoagulação, fotodisrupção, fotoablação ou fotoquímica, são determinadas pelo comprimento de onda juntamente com o tempo de exposição, densidade de potência e densidade de energia. Tais características determinam a absorção da energia que é diferente entre cada tecido do organismo.

Enquanto os meios refrativos transparentes do olho - córnea, cristalino e vítreo, não absorvem radiação magnética no espectro visível de luz, em densidades maiores de energia ocorre absorção com formação de plasma, fenômeno denominado fotodisrupção tecidual. Tal fenômeno ocorre especialmente em comprimentos de onda na faixa próxima ao infravermelho (NIR - near infra-red). A primeira aplicação prática de lasers NIR na Oftalmologia foi com Nd:Yag laser, introduzido por Aron-Rosa no início dos anos $80^{(3)}$. O Nd:Yag laser é amplamente utilizado para realização de abertura na cápsula posterior (capsulotomia) e na íris (iridotomia), bem como para lise de traves vítreas e rotura da hialóide em casos de hemorragia pré-retiniana. Todas estas aplicações determinaram uma diminuição significativa na necessidade de intervenções intraoculares que são inquestionavelmente mais traumáticas e com maior risco. O Nd:YAG laser produz fotodisrupção tecidual em seu ponto de foco, resultando em uma nuvem de elétrons livres e moléculas ionizadas (plasma) que se expande rapidamente. Tipicamente, o Nd:YAG laser tem duração de pulso na faixa de nanosegundo ( $10^{-9}$ do segundo). Nesta faixa de exposição, há formação de ondas acústicas de choque que se dissipam aos tecidos circundantes, tipicamente com zona de dano tecidual de $100 \mu \mathrm{m}(0,1 \mathrm{~mm})$. Tal fato pode ser facilmente percebido quando o feixe de laser atinge a lente intraocular durante a realização de capsulotomia posterior, o que é indesejado, mas gera poucos efeitos colaterais para o paciente. Entretanto, tal volume de dano colateral tecidual tornou impraticável o uso do Nd:Yag laser em cirurgias de córnea, pois estas demandam uma precisão dezenas de vezes maior. Reduzindo a duração dos pulsos de laser com este comprimento de onda (NIR) para a faixa de femtossegundo $\left(10^{-15}\right.$ do segundo), é possível concentrar a energia em uma área cem vezes menor ou submicrônica, o que aumenta significativamente o controle e possibilita cirurgias na córnea.

A primeira observação da interação de um laser NIR de femtossegundo (fs)* com a córnea foi feita por Ronald Kurtz em 1992, quando ainda residente na Universidade de Michigan (Kellogg Eye Center). Kurtz observou um corte limpo e perfeitamente bem delineado na córnea de um cientista que havia tido uma lesão acidental com um laser que estava sendo estudado para indústria automobilística. Juntamente com os engenheiros húngaro Tibor Juhasz e francês

(*) Femtossegundo (fs) é uma unidade de medida de tempo. Corresponde a $10^{-15}$ segundos, ou seja, um milionésimo de um bilionésimo de segundo. O femtossegundo está para um segundo como um segundo está para 32 milhões de anos. (Fonte: www.inmetro.gov.br) 
Gerard Mourou, Kurtz iniciou estudos para as aplicações do laser de femtossegundo na oftalmologia no CUOS (Center for Ultrafast Optical Science) da Universidade de Michigan, de modo a fundar a corporação Intralase em $1997^{(4)}$. Os primeiros aparelhos de laser NIR de femtossegundo, que trabalhavam numa frequência inferior a $10 \mathrm{kHz}$, foram testados na Hungria. O Intralase foi o primeiro laser de femtossegundo NIR a ser aprovado pelo FDA (Food and Drug Adminsitration) americano para realização do corte lamelar do LASIK em $2000 \mathrm{com}$ isenção $510 \mathrm{k}$, ou seja, sem necessidade de estudos por ser comparável ao microcerátomo. O Intralase (Abbot Medical Optics) tornou-se disponível para o mercado internacional em 2001, com uma crescente e rápida aceitação no mercado de cirurgia refrativa. Rapidamente, outros lasers de femtossegundo foram introduzidos num mercado de crescimento rápido e competitivo no qual todas as maiores empresas ligadas a Oftalmologia estão bem representadas (quadro 1).

\section{Quadro 1}

\section{Lasers de Femtossegundo no mercado internacional}

\begin{tabular}{ll}
\hline Intralase & Abbot Medical Optics \\
LDV & Ziemmer Opthalmic \\
Visumax & Zeiss-Meditec \\
Femtec & Technolas Perfect Visuion \\
FS 200 & Alcon-Wavelight \\
LenSx & Alcon \\
LensAR & LensAR \\
CataLYS & OptiMedica \\
\hline
\end{tabular}

Observou-se uma importante evolução nos lasers de femtossegundo, o que se reflete na taxa de repetição, no controle da energia e na capacidade de programar cortes de forma mais versátil na córnea. Por exemplo, o Intralase evolui em cinco gerações até o sistema iFS com 150kHz. A maior velocidade é facilmente apreciada se compararmos o tempo necessário para confecção do flap no LASIK: mais de 1 minuto com o fs $15 \mathrm{kHz}$ X menos de 10 segundos com o iFS $150 \mathrm{kHz}$. Além de maior velocidade, existe maior controle da energia e menor resposta inflamatória. Adicionalmente, é possível se ajustar diversos parâmetros, como a criação de discos ovais e o ângulo de entrada invertido além de $90^{\circ}$ até $150^{\circ}$, o que reduz a chance de crescimento epitelial da interface e proporciona maior estabilidade biomecânica pós-operatória (figura 1A e B).

Diversas são as vantagens do laser de femtossegundo sobre os microcerátomos ${ }^{(5)}$. É verdade que os microcerátomos evoluíram significativamente desde os primeiros modelos adaptados da ceratomileusis in situ como o ACS (Automatic Corneal Shaper). Entretanto, o controle da confecção do corte com o laser reduz o risco de complicações que podem ocorrer ao se criar o flap, como perfuração da lamela (buttom hole), corte irregular e corte incompleto ${ }^{(6)}$. A geometria planar do flap e a maior consistência no controle da espessura do corte reduzem significativamente o risco de cortes mais profundos, que estão associados a um maior risco de desestabilização biomecânica e evolução para ectasia. A maior estabilidade pós-operatória do flap criado pelo femtossegundo está também relacionada com o seu encaixe mais perfeito no leito (figura 1C). Adicionalmente, o impacto do LASIK assistido por laser de femtossegundo é inferior ao realizado pelo microcerátomo na superfície ocular, havendo menor incidência de olho seco e epiteliopatia neurotrófica ${ }^{(7-8)}$.
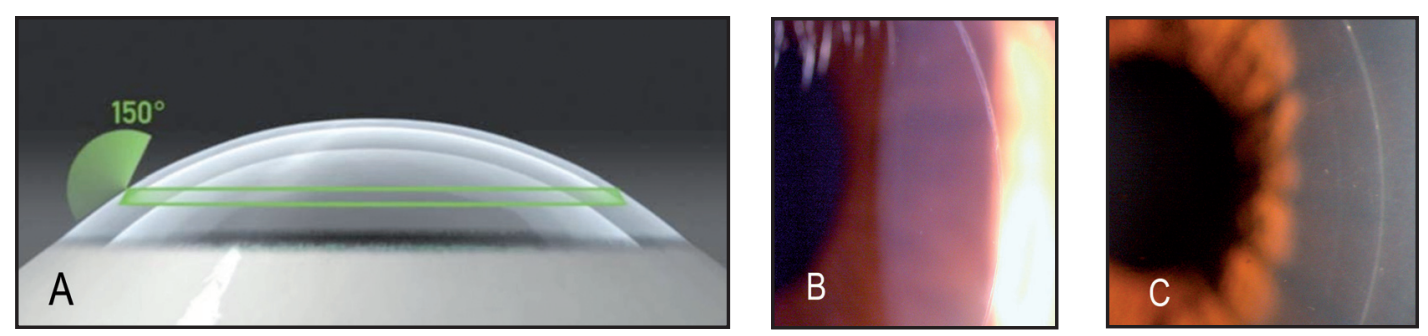

Figura 1:A) Controle da angulação do corte do flap para LASIK; B) Pós-operatório (1 dia) de LASIK assistido por Intralase iFS com angulação de $130^{\circ}$; Note a dupla linha que marca o bordo do flap e limite de dissecção interno do corte angulado; C) Alinhamento do flap no LASIK com Intralase, com exata concordância dos nervos periféricos 
Além da confecção do flap no LASIK, outras aplicações para cirurgia de córnea vêm aumentando a aplicabilidade do laser de femtossegundo. Destacam-se a tunelização para implantes de segmentos de anel estromal (figura 2) ${ }^{(9)}$, biópsia de córnea, ceratotomia incisional para astigmatismo ${ }^{(10)}$, ceratoplastia lamelar ${ }^{(11)}$ e penetrante. ${ }^{(11-13)}$.O laser de femtossegundo pode criar cortes para extração de lentículas, da mesma forma que a ceratomileusis in situ descrita pelo prof. José I. Barraquer na década de 50. O procedimento denominado SMILE (small incision lenticule extraction) foi proposto por Sekundo com o Visumax (Zeiss-Meditec) ${ }^{(14)}$. Outras técnicas revisitadas pelo advento do femtossegundo são as ceratotomias incisionais. Por exemplo, a técnica Intracor, que consiste na realização de incisões circulares concêntricas no estroma para indução de multifocalidade na tentativa de tratamento da presbiopia, foi proposta por Ruiz e Holzer como o Femtec (Technolas Perfect Vision) ${ }^{(15)}$.
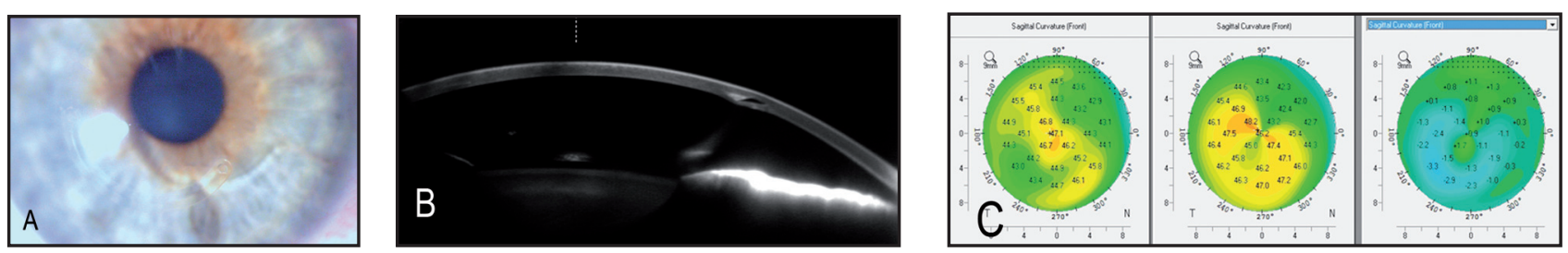

Figura 2: A) Pós-operatório (1 mês) de implante de segmento de anel estromal (Keraring) com iFS. B) Imagem de Scheimpflug com Pentacam; C) Mapa de curvatura axial ou sagital do estado pós, pré-operatório e diferencial deste caso. Observar a correção do padrão irregular do ceratocone em padrão de "C" deitado ou "pata de carangueijo". A acuidade visual sem correção passou de 20/200 para 20/ 30 e a corrigida com óculos de 20/40 para 20/25+, com redução significativa do astigmatismo

Novas plataformas de lasers de femtossegundo permitem aplicação da energia em planos mais profundos, podendo alcançar a cavidade vítrea. Adicionalmente, a integração de sistemas de imagem de Scheimpflug ou OCT (Optical Coherence Tomography) permitem controle da cirurgia durante o procedimento. Com tais avanços, o laser de femtossegundo vem sendo utilizado para facilitar a cirurgia da catarata ${ }^{(16-17)}$. Por outro lado, a realização de incisões no cristalino para recuperação da capacidade acomodativa foi descrita por Krueger, com resultados promissores ${ }^{(18)}$.

Não é necessário uma mente muito inovadora para pensar numa cirurgia de catarata "ideal" assistida por laser de femtossegundo. A fragmentação do cristalino controlada pelo sistema de imagem em tempo real vai aumentar a segurança e reduzir consideravelmente a energia necessária para a sua retirada. A confecção da capsulorrexe será perfeitamente controlada para centralização e tamanho ideais, de acordo com imagens obtidas no pré-operatório, que medem precisamente o diâmetro branco-a-branco e os eixos visuais, de modo a permitir o posicionamento da LIO (lente intraocular) perfeito. A incisão em córnea clara terá uma arquitetura inovadora de modo a reduzir a chance de vazamento e o risco de endoftalmite (figura 3). Incisões estromais para astigmatismo poderão ser confeccionadas, aliando a precisão do laser com os dados dos exames da córnea, incluindo a topografia, tomografia e caracterização biomecânica ${ }^{(19)}$. Finalmente um flap planar pode ser confeccionado de modo a ser utilizado apenas se houver necessidade de um tratamento refrativo com excimer laser personalizado (LASIK) em segundo tempo.

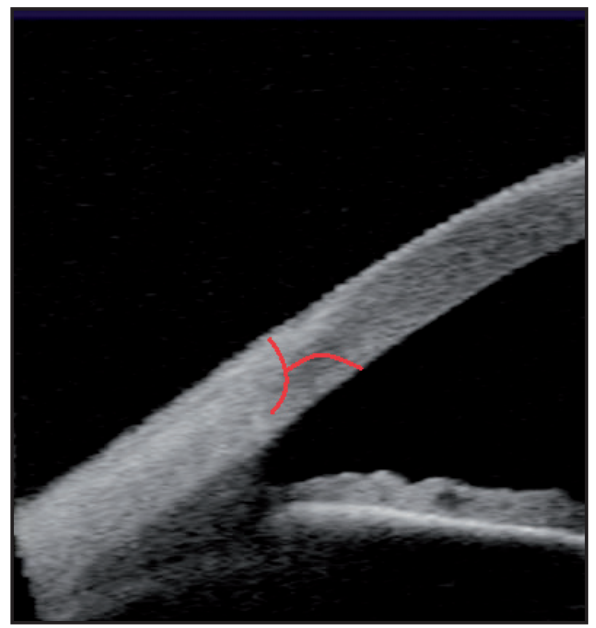

Figura 3: Proposta de incisão em córnea clara com arquitetura em dobradiça angulada para cirurgia de catarata assistida por laser de femtossegundo 
Diferentes plataformas de laser de femtossegundo se especializarão em cirurgias da córnea e da catarata. Entretanto, se uma plataforma for capaz de combinar as aplicações na córnea com, ao menos a realização da capsulorrexe, esta deve ter significativas vantagens no mercado.

Os limites da aplicação do laser de femtossegundo não ficam no cristalino. Por exemplo, McDonnel e colaboradores estudam uma vitreólise em pacientes com retinopatia diabética de modo a evitar a proliferação tracional. Diversas vantagens do laser de femtossegundo sobre as respectivas técnicas manuais vêm sendo descritas ${ }^{(4)}$. Não há dúvidas que os sistemas de laser de femtossegundo já revolucionaram a cirurgia de córnea, trazendo novos patamares de segurança, eficácia para diversos procedimentos com indicações extremamente amplas. Entretanto, os custos elevados desta nova tecnologia devem ser considerados, sendo um verdadeiro desafio disponibilizar tal tecnologia em nosso meio. Certamente a união de grupos de especialistas com o objetivo de oferecer qualidade e segurança para seus pacientes, com a criação de centros de tecnologia, é a solução mais certa para tornar possível a disseminação dos lasers de femtossegundo em nosso país!

\section{Renato Ambrósio Júnior \\ Professor Associado do Programa de pós-graduação em Oftalmologia da Universidade Federal de São Paulo - UNIFESP - São Paulo (SP), Brasil; e da Pontifícia Universidade Católica do Rio de Janeiro - PUC-Rio - Rio de Janeiro (RJ), Brasil; Grupo de Estudos de Tomografia e Biomecânica de Córnea - Rio de Janeiro (RJ), Brasil; Refracta-RIO - Rio de Janeiro (RJ), Brasil; FemtoLaser-RIO - Rio de Janeiro (RJ), Brasil; Instituto de Olhos Renato Ambrósio - Rio de Janeiro (RJ), Brasil}

Endereço para correspondência:
Instituto de Olhos Renato Ambrósio
Rua Conde de Bonfim, $\mathrm{n}^{\circ} 211 / 712-$ Tijuca
CEP 20520-050 - Rio de Janeiro - (RJ), Brasil
Tel/Fax: 55 (21) 2234-4233/2264-4430

\section{RefERÊNCIAS:}

1. Maiman TH. Stimulated Optical Radiation in Ruby. Nature. 1960;187(4736):493-4.

2. Moo-Young GA. Lasers in ophthalmology. West J Med. $1985 ; 143(6): 745-50$.

3. Aron-Rosa DS, Aron JJ, Cohn HC. Use of a pulsed picosecond Nd: YAG laser in 6,664 cases. J Am Intraocul Implant Soc. 1984;10(1):35-9.

4. Soong HK, Malta JB. Femtosecond lasers in ophthalmology. Am J Ophthalmol. 2009;147(2):189-97 e2.

5. Salomao MQ, Wilson SE. Femtosecond laser in laser in situ keratomileusis. J Cataract Refract Surg. 2010;36(6):1024-32.

6. Ambrosio R, Jr., Wilson SE. Complications of laser in situ keratomileusis: etiology, prevention, and treatment. J Refract Surg. 2001;17(3):350-79.

7. Salomao MQ, Ambrosio R, Jr., Wilson SE. Dry eye associated with laser in situ keratomileusis: Mechanical microkeratome versus femtosecond laser. J Cataract Refract Surg. 2009;35(10):1756-60.

8. Ambrosio R, Jr., Tervo T, Wilson SE. LASIK-associated dry eye and neurotrophic epitheliopathy: pathophysiology and strategies for prevention and treatment. J Refract Surg. 2008;24(4):396-407.

9. Pinero DP, Alio JL, El Kady B, Coskunseven E, Morbelli H, Uceda-Montanes A, et al. Refractive and aberrometric outcomes of intracorneal ring segments for keratoconus: mechanical versus femtosecond-assisted procedures. Ophthalmology. 2009;116(9):1675-87.

10. Kumar NL, Kaiserman I, Shehadeh-Mashor R, Sansanayudh W, Ritenour R, Rootman DS. IntraLase-enabled astigmatic keratotomy for post-keratoplasty astigmatism: on-axis vector analysis. Ophthalmology. 2010;117(6):1228-35 e1.
11. Soong HK, Malta JB, Mian SI, Juhasz T. Femtosecond laserassisted lamellar keratoplasty. Arq Bras Oftalmol. 2008;71(4):601-6.

12. Buratto L, Bohm E. The use of the femtosecond laser in penetrating keratoplasty. Am J Ophthalmol. 2007;143(5):737-42.

13. Yoo SH, Hurmeric V. Femtosecond laser-assisted keratoplasty. Am J Ophthalmol. 2011;151(2):189-91.

14. Sekundo W, Kunert KS, Blum M. Small incision corneal refractive surgery using the small incision lenticule extraction (SMILE) procedure for the correction of myopia and myopic astigmatism: results of a 6 month prospective study. Br J Ophthalmol. 2011;95(3):335-9.

15. Holzer MP, Mannsfeld A, Ehmer A, Auffarth GU. Early outcomes of INTRACOR femtosecond laser treatment for presbyopia. J Refract Surg. 2009;25(10):855-61.

16. Nagy Z, Takacs A, Filkorn T, Sarayba M. Initial clinical evaluation of an intraocular femtosecond laser in cataract surgery. J Refract Surg. 2009;25(12):1053-60.

17. Mamalis N. Femtosecond laser: The future of cataract surgery? J Cataract Refract Surg. 2011;37(7):1177-8.

18. Reggiani Mello GH, Krueger RR. Femtosecond laser photodisruption of the crystalline lens for restoring accommodation. Int Ophthalmol Clin. 2011;51(2):87-95.

19. Ambrosio R, Jr., Nogueira LP, Caldas DL, Fontes BM, Luz A, Cazal JO, et al. Evaluation of corneal shape and biomechanics before LASIK. Int Ophthalmol Clin. 2011;51(2):11-38. 